\title{
O capim-marandu baixo no início do diferimento melhora a morfologia do pasto e aumenta o desempenho dos ovinos no inverno
}

[The low marandu palisadegrass at the beginning of stockpiling improves pasture morphology and increases sheep performance in winter]

L.E.F. Afonso ${ }^{1}$, M.E.R. Santos ${ }^{2 *}$, S.P. Silva ${ }^{2}$, A.C. Rêgo ${ }^{1}$, D.M. Fonseca ${ }^{3}$, B.H.R. Carvalho ${ }^{2}$

1Universidade Federal Rural da Amazônia - Belém, PA

Universidade Federal de Uberlândia - Uberlândia, MG

${ }^{3}$ Universidade Federal de Viçosa - Viçosa, MG

\begin{abstract}
RESUMO
Objetivou-se avaliar os efeitos de quatro alturas $(15,25,35$ e $45 \mathrm{~cm})$ da Urochloa brizantha cv. Marandu (capim-marandu) no início do diferimento sobre a morfologia do pasto, a seletividade e o desempenho de ovinos no início, meio e fim do período de pastejo, no inverno. Foi utilizado o delineamento inteiramente ao acaso, com três repetições. Os pastos diferidos com 35 e $45 \mathrm{~cm}$ apresentaram maiores massas de forragem do que aqueles diferidos com 15 e $25 \mathrm{~cm}$. O pasto diferido com $15 \mathrm{~cm}$ apresentou maior porcentagem de folha e menor percentual de colmo morto, em comparação aos demais pastos. A seleção por folha morta aumentou no período de pastejo. Em geral, nos pastos diferidos mais altos, os percentuais de folha viva foram menores e os de colmo morto, maiores nas amostras de pastejo simulado. $O$ desempenho dos ovinos foi superior no pasto diferido com $15 \mathrm{~cm}$, intermediário nos pastos diferidos com 25 e $35 \mathrm{~cm}$, e inferior no pasto diferido com $45 \mathrm{~cm}$. A manutenção do capim-marandu com $15 \mathrm{~cm}$ no início do diferimento resulta em pasto com melhor morfologia, otimiza a seletividade e aumenta o desempenho dos ovinos no inverno.
\end{abstract}

Palavras-chave: composição morfológica, massa de forragem, seletividade animal, desempenho animal, Urochloa brizantha syn. Brachiaria brizantha

\begin{abstract}
The objective of this study was to evaluate the effects of four heights (15, 25, 35, and 45cm) of Urochloa brizantha $c v$. Marandu (marandu palisadegrass) at the beginning of the deferment period on the pasture morphology, selectivity, and performance of sheep during the beginning, middle and end of the grazing period in winter. A completely randomized design with three replications was used. Deferred pastures with 35 and $45 \mathrm{~cm}$ presented larger forage masses than those deferred with 15 and $25 \mathrm{~cm}$. Deferred pasture with $15 \mathrm{~cm}$ presented a higher percentage of live leaf and a lower percentage of dead stem. Dead leaf selection increased during the grazing period. In general, in the higher deferred pastures, the percentages of live leaf were lower and those of dead stalk, higher in the samples of simulated grazing. The sheep performance was higher in the $15 \mathrm{~cm}$ pasture, intermediate in the 25 and $35 \mathrm{~cm}$ pastures, and inferior in the $45 \mathrm{~cm}$ pasture. The maintenance of marandu palisadegrass with $15 \mathrm{~cm}$ at the beginning of the deferment period results in deferred pasture with better morphology, optimizes the selectivity and the increase the performance of the sheep in the pasture deferred during the winter.
\end{abstract}

Keywords: morphological composition, forage mass, animal selectivity, animal performance, Urochloa brizantha syn. Brachiaria brizantha

Recebido em 13 de junho de 2017

Aceito em 26 de setembro de 2017

*Autor para correspondência (corresponding author)

E-mail: manoel.rozalino@ufu.br 


\section{INTRODUÇÃO}

O diferimento do uso de pastagens é estratégia de manejo relativamente fácil, de baixo custo e garante suficiente quantidade de massa de forragem na pastagem para alimentação animal durante os meses de inverno (Silva et al., 2016). Contudo, devido ao período relativamente longo de diferimento e às condições climáticas adversas no fim do outono e inverno, o pasto diferido pode apresentar alto percentual de forragem morta e baixo percentual de folha viva (Sousa et al., 2012; Vilela et al., 2012), o que limita o desempenho animal (Santos et al., 2013).

Durante o período de ocupação, no inverno, o percentual de folha viva decresce, mas os percentuais de colmo e de material morto aumentam no pasto diferido (Silva et al., 2016). Essa modificação da estrutura do pasto diferido influencia a seletividade do animal, que passa a consumir menos folha viva e mais folha morta (Santos et al., 2013). Com isso, a qualidade da dieta ingerida varia acentuadamente durante o período de pastejo, com consequências negativas sobre o desempenho dos animais.

Um modo de melhorar a morfologia do pasto diferido consiste na manutenção do dossel forrageiro baixo no início do período de diferimento (Sousa et al., 2012). Assim, há estímulo ao perfilhamento (Santana et al., 2014), isto é, ocorre maior aparecimento de perfilhos jovens no pasto, os quais têm alta relação folha/colmo. Dessa forma, o pasto diferido com menor altura pode ter morfologia mais favorável à seletividade do animal e, consequentemente, pode incrementar o desempenho animal em pastagens diferidas durante o inverno.

Objetivou-se compreender como a variação da altura do pasto no início do período de diferimento modifica a estrutura da Brachiaria brizantha cv. Marandu syn. Urochloa brizantha cv. Marandu, bem como a seletividade aparente e o desempenho de ovinos durante o inverno.

\section{MATERIAL E MÉTODOS}

O experimento foi conduzido de acordo com as normas éticas e aprovado pela Comissão de Ética no Uso de Animais (Ceua) da Faculdade de Medicina Veterinária da Universidade Federal de Uberlândia (UFU), sob protocolo número 31/13.

O estudo foi realizado de janeiro a setembro de 2013, na Fazenda Experimental Capim-Branco, na UFU, em Uberlândia, MG, Brasil (18³0'S, $\left.47^{\circ} 50^{\prime} \mathrm{W}\right)$. O local do experimento tem altitude de $863 \mathrm{~m}$, está inserido no bioma Cerrado, e seu clima é classificado como Aw, isto é, tropical de altitude (Alvares et al., 2013), com estações seca (de abril a setembro) e chuvosa (de outubro a março) bem definidas. Durante o experimento, as informações sobre as condições climáticas foram monitoradas (Tab. 1).

Tabela 1. Temperatura média do ar, radiação solar média, precipitação e evapotranspiração mensais durante o período experimental

\begin{tabular}{|c|c|c|c|c|c|c|}
\hline \multirow{2}{*}{ Período } & \multicolumn{3}{|c|}{ Temperatura média do ar $\left({ }^{\circ} \mathrm{C}\right)$} & \multirow{2}{*}{$\begin{array}{c}\text { Radiação } \\
\text { solar } \\
\text { (Mj/dia) }\end{array}$} & \multirow{2}{*}{$\begin{array}{l}\text { Precipitação } \\
\text { pluvial (mm) }\end{array}$} & \multirow{2}{*}{$\begin{array}{c}\text { Evapotranspiração } \\
(\mathrm{mm})\end{array}$} \\
\hline & Média & Mínima & Máxima & & & \\
\hline Jan-mar/2013 ${ }^{1}$ & 22,73 & 18,75 & 28,68 & 245,75 & 152,35 & 39,73 \\
\hline Abr-jun/2013 ${ }^{2}$ & 20,70 & 15,67 & 27,07 & 413,37 & 68,73 & 66,47 \\
\hline Jul-set $/ 2013^{3}$ & 20,73 & 14,58 & 28,03 & 495,45 & 9,35 & 84,68 \\
\hline
\end{tabular}

${ }^{1}$ Período antecedente ao diferimento; ${ }^{2}$ período de diferimento; ${ }^{3}$ período de pastejo.

O experimento ocorreu em pastagem com Brachiaria brizantha cv. Marandu syn. Urochloa brizantha cv. Marandu (capim-marandu). Foram utilizados 12 piquetes (unidades experimentais) com $800 \mathrm{~m}^{2}$, além de uma área reserva, totalizando aproximadamente dois hectares.

No início do experimento, foi retirada uma amostra de solo na camada de $0-20 \mathrm{~cm}$, que apresentou os seguintes resultados: $\mathrm{pH}\left(\mathrm{H}_{2} \mathrm{O}\right)$ : 6,$1 ; \quad \mathrm{P}: \quad 4,5 \mathrm{mg} / \mathrm{dm}^{3} \quad$ (Mehlich-1); $\quad \mathrm{K}^{+}$: $138,8 \mathrm{mg} / \mathrm{dm}^{3} ; \quad \mathrm{Ca}^{2+}: \quad 5,5 \mathrm{cmol}_{\mathrm{c}} / \mathrm{dm}^{3} ; \quad \mathrm{Mg}^{2+}$ : $1,9 \mathrm{cmol}_{\mathrm{c}} / \mathrm{dm}^{3} ; \mathrm{Al}^{3+}: 0,0 \mathrm{cmol}_{\mathrm{c}} / \mathrm{dm}^{3}(\mathrm{KCl} 1 \mathrm{~mol} / \mathrm{L})$; CTC efetiva: 7,3; CTC a pH 7,0: 10,2; e saturação por bases: $72,0 \%$. Com base nesses resultados e de acordo com as recomendações de Cantarutti et al. (1999) para um sistema de médio nível tecnológico, foram aplicados 
$50 \mathrm{~kg} / \mathrm{ha}$ de $\mathrm{P}_{2} \mathrm{O}_{5}$ e de $\mathrm{N}$ em janeiro, além de $70 \mathrm{~kg} / \mathrm{ha}$ de $\mathrm{N}$ em março. Como fontes de adubos, foram utilizados o superfosfato simples e a ureia.

O experimento foi conduzido em delineamento inteiramente ao acaso, com três repetições. Foram avaliadas quatro alturas do pasto $(15,25$, 35 e $45 \mathrm{~cm}$ ) no início do período de diferimento. Após o período de diferimento, todos os pastos foram avaliados, com medidas repetidas no tempo, durante o início (sétimo dia), o meio $\left(45^{\circ}\right.$ dia) e o fim $\left(90^{\circ}\right.$ dia) do período de pastejo.

De janeiro a março, todos os pastos foram manejados com ovinos em lotação contínua e com taxa de lotação variável para manter as alturas médias almejadas, de acordo com os tratamentos. O critério usado para mensuração da altura do pasto consistiu da distância desde a superfície do solo até a folha viva mais alta do dossel. A altura dos pastos foi mensurada semanalmente e controlada com adição ou retirada de animais nos piquetes. O período de diferimento, no qual os piquetes ficaram sem animais, foi de 79 dias, com início em 03/04/2013. Após o período de diferimento, iniciou-se o período de pastejo (90 dias) de todos os pastos. Estes foram manejados em lotação contínua e taxa de lotação fixa, com ovinos mestiços Santa Inês x Dorper, com quatro meses de idade e peso médio inicial de $26 \mathrm{~kg}$. A alocação dos animais nos piquetes foi feita de forma aleatória, para manter quatro ovinos por piquete, o que correspondeu a 2,8UA/ha.

Todas as avaliações dos pastos diferidos foram realizadas no início (sétimo dia), no meio $\left(45^{\circ}\right.$ dia) e no fim $\left(90^{\circ}\right.$ dia) do período de pastejo. Em cada piquete e em três áreas com a mesma altura média do pasto, foi realizado o corte ao nível da superfície do solo de todos os perfilhos contidos no interior de um quadrado de $0,25 \mathrm{~m}^{2}$, constituindo-se uma amostra. Esta foi pesada e subdividida em duas subamostras. Uma delas foi separada em folha viva, colmo vivo, folha morta e colmo morto. As partes do colmo e da lâmina foliar com amarelecimento e, ou, necrosamento foram incorporadas às frações colmo morto e folha morta, respectivamente. Após a separação, os componentes foram pesados e secos em estufa de circulação forçada de ar a $65^{\circ} \mathrm{C}$, por 72 horas. A partir desses dados, estimaram-se as porcentagens de cada componente morfológico na forragem disponível. A outra subamostra foi pesada, seca nas mesmas condições anteriores e novamente pesada, para estimar a massa de forragem do pasto.

O método de simulação de pastejo animal foi usado para coletar uma amostra de forragem por piquete. Cada amostra foi separada em folha viva, colmo vivo, folha morta e colmo morto, adotando-se os mesmos critérios descritos anteriormente. Cada subamostra foi seca em estufa com ventilação forçada, a $65^{\circ} \mathrm{C}$, durante 72 horas, e pesada, para obtenção da sua composição morfológica, em porcentagem.

A seletividade aparente exercida pelos animais em relação aos diferentes componentes morfológicos do pasto foi avaliada com base na seguinte equação (Santos et al., 2016): ISA = SP/FD, em que: ISA = índice de seletividade aparente; $\mathrm{SP}=$ componente morfológico na amostra de simulação de pastejo (\%); FD = componente morfológico na amostra de forragem disponível (\%).

Durante o período de pastejo, no inverno, todos os animais foram vermifugados a cada 30 dias. $\mathrm{O}$ desempenho dos animais foi obtido pela diferença de peso médio dos animais entre o fim e o início do período de pastejo, cujo valor foi dividido pelo período de pastejo. A produção animal por unidade de área da pastagem, expressa em $\mathrm{kg} / \mathrm{ha}$.dia, foi calculada pelo produto entre o desempenho animal e o número de animais nos piquetes.

Para análise dos dados, inicialmente, aqueles que não atenderam aos pressupostos da análise de variância (aditividade, independência dos erros, normalidade dos erros e homogeneidade de variância dos erros) foram transformados. Depois, para as características do pasto e da seletividade animal, procedeu-se à análise de variância em delineamento inteiramente ao acaso, usando-se o Proc Mixed do SAS. Os efeitos de altura e período de pastejo e sua interação foram considerados fixos. Os dias do período de pastejo foram considerados medidas repetidas no tempo. Para análise dos dados de desempenho animal e de produção animal por área de pastagem, foi utilizado o procedimento GLM do SAS. As médias dos fatores foram comparadas pelo teste de Tukey $(\mathrm{P}<0,05)$. 


\section{RESULTADOS E DISCUSSÃO}

Entre as características do pasto e da seletividade dos ovinos avaliadas, oito foram influenciadas $(\mathrm{P}<0,05)$ de forma isolada pela altura do pasto (Tab. 2) e pelo período de pastejo (Tab. 3). Por outro lado, as porcentagens de colmo vivo e de folha morta na forragem disponível, o percentual de folha morta na amostra de simulação de pastejo e os índices de seletividade aparente das folhas viva e morta foram influenciados pela interação entre a altura do pasto e o período de pastejo (Tab. 4).

Tabela 2. Características do capim-marandu, das amostras de simulação de pastejo e índice de seletividade aparente (ISA) de ovinos em pastagens diferidas com variação de altura do pasto no início do período de diferimento

\begin{tabular}{cccccc}
\hline \multirow{2}{*}{ Característica } & \multicolumn{4}{c}{ Altura do pasto $(\mathrm{cm})$} & \multirow{2}{*}{ EPM } \\
\cline { 2 - 5 } & 15 & 25 & 35 & 45 & 596 \\
Massa de forragem (MF) & $6457 \mathrm{c}$ & $7979 \mathrm{~b}$ & $9028 \mathrm{a}$ & $8928 \mathrm{a}$ & 2,3 \\
Folha viva na MF (\%) & $20,4 \mathrm{a}$ & $13,0 \mathrm{~b}$ & $10,5 \mathrm{~b}$ & $10,6 \mathrm{~b}$ & 2,7 \\
Colmo morto na MF (\%) & $15,9 \mathrm{~b}$ & $25,8 \mathrm{a}$ & $27,5 \mathrm{a}$ & $26,5 \mathrm{a}$ & 2,8 \\
Folha viva na SP (\%) & $55,2 \mathrm{a}$ & $56,7 \mathrm{a}$ & $46,5 \mathrm{~b}$ & $45,8 \mathrm{~b}$ & 0,3 \\
Colmo vivo na SP (\%) & $6,2 \mathrm{a}$ & $4,8 \mathrm{a}$ & $4,9 \mathrm{a}$ & $5,8 \mathrm{a}$ & 1,4 \\
Colmo morto na SP (\%) & $5,4 \mathrm{~b}$ & $5,0 \mathrm{~b}$ & $10,7 \mathrm{a}$ & $9,3 \mathrm{a}$ & 0,01 \\
ISA do colmo vivo & $0,20 \mathrm{a}$ & $0,16 \mathrm{a}$ & $0,13 \mathrm{a}$ & $0,17 \mathrm{a}$ & 0,03 \\
ISA do colmo morto & $0,26 \mathrm{ab}$ & $0,19 \mathrm{~b}$ & $0,35 \mathrm{a}$ & $0,31 \mathrm{a}$ & 0,03 \\
\hline
\end{tabular}

SP: amostra de simulação de pastejo animal; EPM: erro-padrão da média. Para cada característica, médias seguidas por letras diferentes diferem pelo teste de Tukey $(\mathrm{P}<0,05)$.

A massa de forragem (MF) no fim do período de diferimento é resultado da MF no início desse período, acrescida da produção de forragem durante o diferimento. Como existe associação positiva entre a MF e a altura do pasto (Paula et al., 2012; Nantes et al., 2013), é natural o incremento da MF com o aumento da altura do pasto no início do diferimento (Tab. 2), conforme observaram Vilela et al. (2013) e Silva et al. (2015) em trabalhos com gramíneas do gênero Brachiaria. Além disso, no pasto mais alto, a competição por luz é alta (Carnevalli et al., 2006) e pode ter resultado na morte dos perfilhos menores, que ficam sombreados pelos maiores, o que pode justificar o maior percentual de colmo morto nos pastos diferidos com 25, 35 e $45 \mathrm{~cm}$, em relação àquele diferido com $15 \mathrm{~cm}$ (Tab. 2).

Os perfilhos dos pastos com maior altura inicial $(35$ e $45 \mathrm{~cm})$ tendem a passar por maior competição intraespecífica por luz durante o período de diferimento e, consequentemente, alongam seu colmo para expor as folhas jovens no estrato mais alto no dossel, onde a luminosidade é maior (Carnevalli et al., 2006). Esse fato justifica o maior percentual de colmo vivo em pastos diferidos mais altos (Tab. 4), o que pode dificultar o pastejo dos animais (Benvenutti et al., 2008). Todavia, o percentual de colmo vivo na MF reduziu no fim, em comparação ao início e ao meio do período de pastejo (Tab. 4), possivelmente pela mortalidade de grande parte dos perfilhos, causada pelo clima restritivo à planta no inverno (Tab. 1).

O menor sombreamento no interior do dossel diferido com $15 \mathrm{~cm}$ provavelmente ocasionou menor senescência e, com efeito, maior percentual de folha viva nesse pasto, quando comparado aos demais (Tab. 2). Esse mesmo padrão de resposta foi observado por Santos et al. (2013), que avaliaram quatro alturas (10, 20, 30 e $40 \mathrm{~cm}$ ) do pasto de Urochloa decumbens cv. Basilisk syn. Brachiaria decumbens cv. Basilisk no início do diferimento.

A inferior competição por luz no dossel forrageiro diferido com $15 \mathrm{~cm}$ também justifica o seu menor percentual de folha morta no início do período de pastejo, em relação aos demais pastos (Tab. 4). Contudo, no meio e, principalmente, no fim do período de pastejo, os pastos diferidos mais baixos apresentaram, em geral, maior percentual de folha morta do que os mais altos (Tab. 4). Provavelmente, grande parte da folha viva do pasto diferido mais baixo senesceu no meio e no fim do período de pastejo, e isso aumentou o seu percentual de folha morta. Por outro lado, é possível que a menor quantidade de folha viva dos pastos diferidos mais altos tenha 
limitado o pastejo seletivo dos ovinos por esse componente morfológico (Santos et al., 2013).

Dessa forma, os animais passaram a consumir mais folha morta, o que reduziu seu percentual durante o meio e o fim do período de pastejo dos pastos altos (Tab. 4).

Tabela 3. Características do capim-marandu, das amostras de simulação de pastejo e índice de seletividade aparente (ISA) de ovinos durante o período de pastejo, no inverno, em pastagens diferidas

\begin{tabular}{ccccc}
\hline Característica & \multicolumn{3}{c}{ Período de pastejo } & \multirow{2}{*}{ EPM } \\
\cline { 2 - 4 } & Início & Meio & Fim & \\
\hline Massa de forragem (MF) & $9568 \mathrm{a}$ & $8381 \mathrm{~b}$ & $6344 \mathrm{c}$ & 941 \\
Folha viva na MF (\%) & $30,1 \mathrm{a}$ & $9,8 \mathrm{~b}$ & $1,0 \mathrm{c}$ & 8,6 \\
Colmo morto na MF (\%) & $17,0 \mathrm{~b}$ & $26,8 \mathrm{a}$ & $28,1 \mathrm{a}$ & 3,5 \\
Folha viva na SP (\%) & $88,7 \mathrm{a}$ & $58,5 \mathrm{~b}$ & $6,0 \mathrm{c}$ & 24,2 \\
Colmo vivo na SP (\%) & $7,5 \mathrm{a}$ & $2,7 \mathrm{~b}$ & $6,2 \mathrm{c}$ & 1,4 \\
Colmo morto na SP (\%) & $1,2 \mathrm{c}$ & $4,4 \mathrm{~b}$ & $17,2 \mathrm{a}$ & 4,9 \\
ISA do colmo vivo & $0,21 \mathrm{a}$ & $0,08 \mathrm{~b}$ & $0,21 \mathrm{a}$ & 0,04 \\
ISA do colmo morto & $0,07 \mathrm{c}$ & $0,17 \mathrm{~b}$ & $0,60 \mathrm{a}$ & 0,2 \\
\hline
\end{tabular}

SP: amostra de simulação de pastejo animal; EPM: erro-padrão da média. Para cada característica, médias seguidas por letras diferentes diferem pelo teste de Tukey $(\mathrm{P}<0,05)$.

Tabela 4. Características dos pastos de capim-marandu, de amostras de simulação de pastejo e índice de seletividade aparente de ovinos durante o período de pastejo em pastagens com alturas variáveis no início do diferimento

\begin{tabular}{|c|c|c|c|c|c|}
\hline \multirow{3}{*}{$\begin{array}{l}\text { Período de } \\
\text { pastejo }\end{array}$} & \multicolumn{4}{|c|}{ Altura $(\mathrm{cm})$} & \multirow{3}{*}{ EPM } \\
\hline & 15 & 25 & 35 & 45 & \\
\hline & \multicolumn{4}{|c|}{ Colmo vivo (\%) na forragem disponível } & \\
\hline Início & $33,1 \mathrm{bA}$ & $31,2 \mathrm{bA}$ & $38,0 \mathrm{aA}$ & $40,3 \mathrm{aA}$ & \multirow{3}{*}{1,1} \\
\hline Meio & $33,7 \mathrm{bA}$ & $34,0 \mathrm{bA}$ & 37,9 aA & $34,2 \mathrm{bB}$ & \\
\hline Fim & $29,4 \mathrm{bB}$ & $28,1 \mathrm{bB}$ & $33,4 \mathrm{aB}$ & $29,2 \mathrm{bC}$ & \\
\hline \multicolumn{6}{|c|}{ Folha morta (\%) na forragem disponível } \\
\hline Início & $15,9 \mathrm{bC}$ & $19,6 \mathrm{aC}$ & $19,5 \mathrm{aC}$ & $20,1 \mathrm{aC}$ & \multirow{3}{*}{2,9} \\
\hline Meio & $32,6 \mathrm{aB}$ & $26,8 \mathrm{bB}$ & $25,2 \mathrm{bB}$ & $28,1 \mathrm{bB}$ & \\
\hline Fim & $46,9 \mathrm{aA}$ & $44,5 \mathrm{aA}$ & $34,3 \mathrm{bA}$ & $37,9 \mathrm{bA}$ & \\
\hline \multicolumn{6}{|c|}{ Folha morta (\%) na amostra de simulação de pastejo } \\
\hline Início & $2,0 \mathrm{cC}$ & $2,0 \mathrm{cC}$ & $3,5 \mathrm{aC}$ & $2,9 \mathrm{bC}$ & \multirow{3}{*}{8,5} \\
\hline Meio & $26,3 \mathrm{bB}$ & $25,5 \mathrm{bB}$ & $40,8 \mathrm{aB}$ & $44,8 \mathrm{aB}$ & \\
\hline Fim & $71,2 \mathrm{aA}$ & $72,9 \mathrm{aA}$ & $69,0 \mathrm{aA}$ & 69,8 aA & \\
\hline \multicolumn{6}{|c|}{ Índice de seletividade aparente da folha viva } \\
\hline Início & $2,1 \mathrm{bB}$ & $3,1 \mathrm{aB}$ & $3,5 \mathrm{aC}$ & $3,6 \mathrm{aB}$ & \multirow{3}{*}{0,9} \\
\hline Meio & $4,0 \mathrm{cA}$ & $8,5 \mathrm{aA}$ & $6,9 \mathrm{bB}$ & $7,0 \mathrm{bA}$ & \\
\hline Fim & $4,3 \mathrm{cA}$ & $7,6 \mathrm{bA}$ & $11,8 \mathrm{aA}$ & $2,0 \mathrm{dC}$ & \\
\hline \multicolumn{6}{|c|}{ Índice de seletividade aparente da folha morta } \\
\hline Início & $0,1 \mathrm{aC}$ & $0,1 \mathrm{aC}$ & $0,2 \mathrm{aC}$ & $0,1 \mathrm{aB}$ & \multirow{3}{*}{0,2} \\
\hline Meio & $0,8 \mathrm{bB}$ & $1,0 \mathrm{bB}$ & $1,6 \mathrm{aB}$ & $1,6 \mathrm{aA}$ & \\
\hline Fim & $1,5 \mathrm{bA}$ & $1,6 \mathrm{bA}$ & $2,0 \mathrm{aA}$ & $1,8 \mathrm{bA}$ & \\
\hline
\end{tabular}

EPM: erro-padrão da média. Para cada característica, médias seguidas de mesma letra, minúscula na linha e maiúscula na coluna, não diferem pelo teste de Tukey $(\mathrm{P}>0,05)$. 
Durante o período de pastejo, no inverno, as condições climáticas foram adversas ao crescimento da planta (Tab. 1). Por conseguinte, os ovinos consumiram o pasto diferido, que não rebrotou ou rebrotou muito pouco. Com isso, é natural que a massa de forragem dos pastos tenha reduzido nesse período (Tab. 3). Visto que os ruminantes consomem preferencialmente a folha viva e rejeitam o colmo morto do pasto (Santos et al., 2016), o percentual de folha viva reduziu, enquanto o de colmo morto incrementou durante o período de pastejo (Tab. 3). A redução no percentual de folha viva também foi verificado por Trindade et al. (2007) durante o período de ocupação do pasto de $B$. brizantha $\mathrm{cv}$. Marandu manejada em lotação intermitente e com bovinos.

Como a característica do pasto determina a característica da forragem consumida pelos animais em pastejo (Santos et al., 2016), foram verificados os mesmos padrões de respostas dos percentuais de folha e de colmo morto, tanto no pasto diferido quanto nas amostras de pastejo simulado, de modo que, nos pastos diferidos mais altos, os percentuais de folha viva foram menores e os de colmo morto, maiores (Tab. 2).

$\mathrm{O}$ alto percentual de colmo vivo na amostra de simulação de pastejo (SP) durante o início do período de pastejo (Tab. 3) pode ter sido causado pelo consumo de inflorescência pelos ovinos. Neste trabalho, as inflorescências foram incluídas na fração colmo vivo.

Com o passar do período de pastejo, a morfologia do pasto piorou e a massa de forragem disponível reduziu, e isso dificultou a seleção da folha viva e a rejeição do colmo morto pelos ovinos (Tab. 3). Assim, o animal teve que modificar mais a composição morfológica da dieta ingerida, em relação ao pasto ofertado, o que fez o índice de seletividade aparente (ISA) da folha viva aumentar durante o período de pastejo dos pastos diferidos com 15 , 25 e $35 \mathrm{~cm}$ (Tab. 4). Com o incremento do percentual de colmo morto na amostra de simulação de pastejo, seu ISA também aumentou durante o período de pastejo (Tab. 3).

No pasto diferido com $15 \mathrm{~cm}$, o ISA da folha viva foi menor no início e no meio do período de pastejo, quando comparado aos outros pastos (Tab. 4). Isso indica que o ovino não precisou modificar tanto a forragem aparentemente consumida, em relação às características do pasto disponível; ou seja, pode-se inferir que a morfologia do pasto diferido $\mathrm{com} 15 \mathrm{~cm}$ foi melhor do que a dos demais (Tab. 2). Todavia, no fim de período de pastejo, o pasto diferido com $45 \mathrm{~cm}$ apresentou menor valor de ISA da folha viva, quando comparado aos demais (Tab. 4). É possível que a estrutura do pasto tenha piorado e, assim, dificultado a seleção dos ovinos pela folha viva. Nesse cenário, os animais passam a selecionar mais a folha morta (Tab. 4). Os herbívoros em pastejo tomam uma série de decisões em relação à aceitação ou rejeição dos componentes morfológicos do pasto (Stephens, 2008), para otimizar a coleta de nutrientes de modo rápido e com menor relação custo/benefício. Nesse sentido, embora a folha viva seja de mais fácil apreensão (Benvenutti et $a l ., 2008$ ) e de melhor valor nutritivo (Nave et al., 2010), sua escassez no pasto pode ter aumentado o tempo para que os ovinos a selecionasse, bem como reduzido a massa do bocado dos animais. Com isso, a relação custo/benefício foi desfavorável para seleção da folha viva ao término do período de pastejo, o que levou os ovinos a consumirem mais a folha morta. A folha morta tem maiores percentuais de matéria seca e fibra em detergente neutro (FDN) potencialmente digestiva, bem como inferior percentual de FDN indigestível, em relação ao colmo vivo e ao colmo morto (Santos et al., 2010), o que justificaria o consumo e a seletividade animal pela folha morta quando há escassez de folha viva.

Os pastos diferidos com 35 e $45 \mathrm{~cm}$ apresentaram pior composição morfológica (Tab. 2). Por isso, a porcentagem de folha morta foi maior na amostra de SP coletadas nesses pastos durante o início e o meio do período de pastejo (Tab. 4). Com a deterioração da estrutura do pasto no fim do período de pastejo, caracterizada pela elevação dos percentuais de forragem morta nos pastos (Tab. 3), não ocorreu diferença entre os pastos diferidos para o percentual de folha na amostra de SP (Tab. 4). Salienta-se que, apenas no fim do período de pastejo dos pastos diferidos com 15 e $25 \mathrm{~cm}$, os ovinos selecionaram a folha morta (ISA>1,0). Porém, essa seleção ocorreu mais cedo, durante o meio do período de pastejo nos pastos diferidos com 35 e $45 \mathrm{~cm}$ (Tab. 4). Essa seleção mais precoce dos ovinos pela folha morta nos pastos diferidos mais altos, em 
comparação aos mais baixos, indica que a estrutura dos pastos altos piorou mais rapidamente durante o período de pastejo (Tab. 2).

A melhor morfologia do pasto diferido com $15 \mathrm{~cm}$ (Tab. 2) facilitou o consumo de folha viva pelos animais em pastejo (Tab. 2). Como a folha viva possui melhor valor nutricional (Nave et al., 2010), seu maior consumo pelos animais justificou o superior desempenho dos ovinos nesse pasto (Fig. 1A), em comparação aos demais. Por outro lado, a degradação da morfologia do pasto diferido com $45 \mathrm{~cm}$, que ocorreu mais precocemente durante o período de pastejo, possivelmente limitou a ingestão de forragem pelo ruminante (Fonseca et al., 2012), e isso resultou em menor desempenho dos ovinos (Fig. 1A). Uma vez que a taxa de lotação de todos os pastos diferidos foi fixa durante $o$ experimento, as variações observadas na produção animal por unidade de área (Fig. 1B) foram decorrentes apenas das modificações no desempenho dos animais.
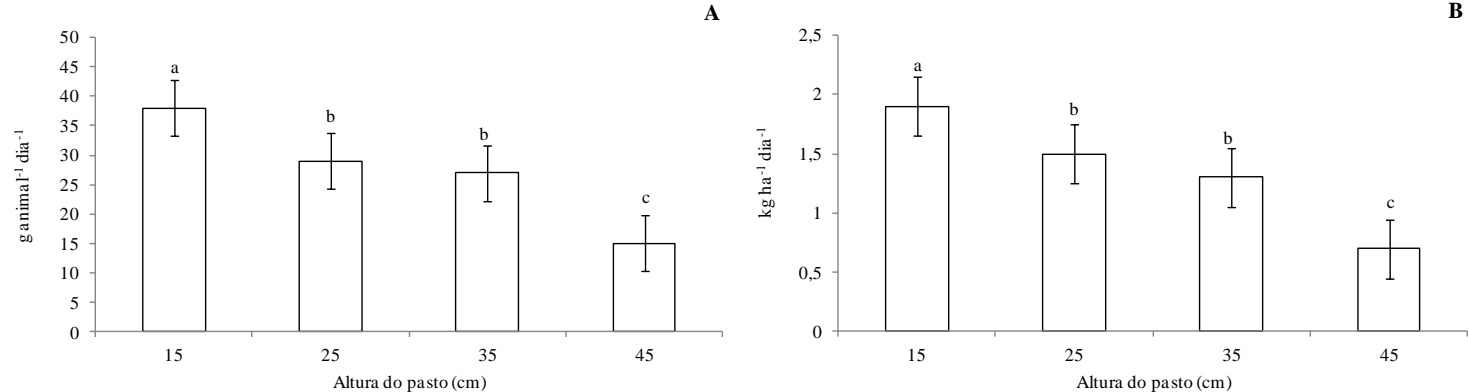

Para cada gráfico, médias seguidas de mesma letra não diferem pelo teste de Tukey $(\mathrm{P}>0,05)$.

Figura 1. Desempenho individual (A) e produção por unidade de área (B) de ovinos durante o inverno em pastagens com capim-marandu com quatro alturas no início do período de diferimento.

Os dados deste trabalho indicam que a redução da altura do pasto no início do período de diferimento é estratégia de manejo adequada para obtenção de pasto diferido com morfologia mais favorável à seletividade e ao desempenho animal durante o inverno. Além disso, durante o período de pastejo, a morfologia do pasto diferido piora e há comprometimento da seletividade animal, um processo que ocorre de modo mais acentuado nos pastos diferidos com maior altura no início do diferimento.

\section{CONCLUSÕES}

A manutenção do capim-marandu com $15 \mathrm{~cm}$ no início do período de diferimento resulta em pasto diferido com melhor morfologia, otimiza a seletividade aparente e aumenta o desempenho dos ovinos na pastagem diferida durante $o$ inverno. A porcentagem de folha viva no pasto diferido diminui durante o período de pastejo no inverno, o que leva os ovinos a consumirem mais a folha morta.

\section{REFERÊNCIAS}

ALVARES, C.A.; STAPE, J.L.; SENTELHAS, P.C. et al. Köppen's climate classification map for Brazil. Met. Z., v.22, p.711-728, 2013.

BENVENUTTI, M.A.; GORDON, I.J.; POPPI, D.P. The effects of stem density of tropical swards and age of grazing cattle on their foraging behaviour. Grass For. Sci., v.63, p.1-8, 2008.

CANTARUTTI, R.B.; MARTINS, C.E.; CARVALHO, M.M. et al. Pastagens. In: RIBEIRO, A.C.; GUIMARÃES, P.T.G.; ALVAREZ V.V.H. (Eds.). Recomendações para $o$ uso de corretivos e fertilizantes em Minas Gerais - $5^{\mathrm{a}}$ aproximação. Viçosa-MG: CFSEMG/UFV, 1999. p.332-341.

CARNEVALLI, R.A.; SILVA, S.C.; BUENO, A.A.O. et al. Herbage production and grazing losses in Panicum maximum cv. Mombaça under four grazing managements. Trop. Grass., v.40, p.165-176, 2006. 
FONSECA, L.; MEZZALIRA, J.C.; BRAMM, C. et al. Management targets for maximising the short-term herbage intake rate of cattle grazing in Sorghum bicolor. Livest. Sci., v.145, p.205-211, 2012.

NANTES, N.N.; EUCLIDES, V.P.B. MONTAGNER, D.B. et al. Desempenho animal e características de pastos de capim-piatã submetidos a diferentes intensidades de pastejo. Pesqui. Agropecu. Bras., v.48, p.114-121, 2013.

NAVE, R.L.G.; PEDREIRA, C.G.S.; PEDREIRA, B.C. Nutritive value and physical characteristics of Xaraes palisadegrass as affected by grazing strategy. S. Afr. J. Anim. Sci., v.40, p.285-293, 2010.

PAULA C.C.L.; EUCLIDES V.P.B.; MONTAGNER D.B. et al. Estrutura do dossel, consumo e desempenho animal em pastos de capim-marandu sob lotação contínua. Arq. Bras. Med. Vet. Zootec., v.64, p.169-176, 2012.

SANTANA, S.S.; FONSECA, D.M.; SANTOS, M.E.R. et al. Initial height of pasture deferred and utilized in winter and tillering dynamics of signal grass during the following spring. Acta Sci. Anim. Sci., v.36, p.17-23, 2014.

SANTOS, M.E.R.; FONSECA, D.M.; BALBINO, E.M. et al. Valor nutritivo de perfilhos e componentes morfológicos em pastos de capim-braquiária diferidos e adubados com nitrogênio. Rev. Bras. Zootec., v.39, p.19191927, 2010.

SANTOS, M.E.R.; FONSECA, D.M.; SOUSA, D.O.C. Seletividade aparente de bovinos em pastos de capim-braquiária sob períodos de diferimento. Arq. Bras. Med. Vet. Zootec., v.68, p.1655-1663, 2016.
SANTOS, M.E.R.; SILVEIRA, M.C.T.; GOMES, V.M. et al. Pasture height at the beginning of deferment as a determinant of signal grass structure and potential selectivity by cattle. Acta Sci. Anim. Sci., v.35, p.379-385, 2013.

SILVA, A.A.S.; FONSECA, D.M.DA.; SANTOS, M.E.R. et al. Initial height and nitrogen fertilization on deferred signalgrass. Biosci. J., v.31, p1671-1681, 2015.

SILVA, C.S.; MONTAGNER, D.B.; EUCLIDES, V.PB. et al. Steer performance on deferred pastures of Brachiaria brizantha and Brachiaria decumbens. Ciênc. Rural, v.46, p.1998-2004, 2016.

SOUSA, B.M.L.; VILELA, H.H.; SANTOS, M.E.R. et al. Characterization of tillers in deferred Piata palisade grass with different initial heights and nitrogen levels. Rev. Bras. Zootec., v.41, 1618-1624, 2012.

STEPHENS, D.W. Decision ecology: foraging and the ecology of animal decision making. Cognit. Affect. Behav. Neurosci., v.8, p.475-484, 2008.

TRINDADE, J.K.; SILVA, S.C.; SOUZA JÚNIOR, S.J. et al. Composição morfológica da forragem consumida por bovinos de corte durante o rebaixamento do capim-marandu submetido a estratégias de pastejo rotativo. Pesqui. Agropecu. Bras., v.42, p.883-890, 2007.

VILELA H.H.; SOUSA, B.M.L.; SANTOS, M.E.R. et al. Characterization of tillers of piata palisade grass deferred in the fall with varying heights and deferment periods. Acta Sci. Anim. Sci., v.35, p.21-27, 2013.

VILELA, H.H.; SOUSA, B.M.L.; SANTOS M.E.R. et al. Forage mass and structure of piatã grass deferred at different heights and variable periods. Rev. Bras. Zootec., v.41, p.1625-1631, 2012. 